\title{
Hysterosalpingographic Findings among Patients Undergoing Infertility Work-Up in Kisangani, Democratic Republic of the Congo
}

\author{
Jean-Didier Bosenge Nguma ${ }^{{ }^{*}}$, Antoine Modia 0'yandjo', Jeanot Juakali Sialikyolo', \\ Gaston Litalema Liogo², Antoine Molua Aundu³, Roger Amisi Kitoko4, \\ Gédeon Katenga Bosunga ${ }^{1}$
}

${ }^{1}$ Department of Gynecology-Obstetrics, Faculty of Medicine and Pharmacy, University of Kisangani, Kisangani, DRC

${ }^{2}$ Department of Medical Imaging, Radiology Unit, Kisangani University Hospital, Kisangani, DRC

${ }^{3}$ Department of Medical Imaging, Unit of Imaging of Women, University of Kinshasa, Kinshasa, DRC

${ }^{4}$ Department of Surgery, Faculty of Medicine and Pharmacy, University of Kisangani, Kisangani, DRC

Email: ^jdidier.bosenge@unikis.ac.cd

How to cite this paper: Bosenge Nguma, J.-D., O'yandjo, A.M., Sialikyolo, J.J., Liogo, G.L., Aundu, A.M., Kitoko, R.A. and Bosunga, G.K. (2019) Hysterosalpingographic Findings among Patients Undergoing Infertility Work-Up in Kisangani, Democratic Republic of the Congo. Open Journal of Obstetrics and Gynecology, 9, 267-277. https://doi.org/10.4236/ojog.2019.92028

Received: February 5, 2019

Accepted: February 25, 2019

Published: February 28, 2019

Copyright $\odot 2019$ by author(s) and Scientific Research Publishing Inc. This work is licensed under the Creative Commons Attribution International License (CC BY 4.0).

http://creativecommons.org/licenses/by/4.0/

\begin{abstract}
Introduction: Hysterosalpingography is an important tool in the investigation of the causes of female infertility. In developing countries such as the Democratic Republic of Congo, the HSG is the basic tool for performing first-line anatomical (pelvic) assessment. The aim of this study is to determine the prevalence of the different lesions observed in hysterosalpingography carried out during the assessment of infertile women monitored in Kisangani. The study set also out to look for differences in these lesions according to the type of infertility. Methods: This cross-sectional study was conducted in Kisangani from June 2016 to December 2018. The 130 cases of hysterosalpingography performed for female infertility assessment during the study period were included, of which $33.07 \%$ for primary infertility and 66.93\% for secondary infertility. Results: The mean age of the patients was $33.52 \pm 6.01$ years; the history of pelvic inflammatory disease was noted with a predominance for secondary infertility ( $11 \%$ vs. $4.65 \%)$. Using hysterosalpingography, 7 (5.38\%) patients had a normal result; 94 (72.31\%) had tubal obstruction; 25 (19.23\%) had hydrosalpinx; 11 (8.46\%) had uterine fibroids; pelvic adhesions were suspected in $12.64 \%$ of cases. There was no obvious association between all these lesions and the type of infertility. Conclusion: We found that tubal obstructions were the most prevalent lesions in this study, regardless of the type of infertility. These lesions would reflect the high prevalence of septic abortions and sexually transmitted infections in developing countries such as the Democratic Republic of the Congo.
\end{abstract}




\section{Keywords}

Hysterosalpingography, Female Infertility, Tubal Obstruction, Hydrosalpinx, Pelvic Adhesion, Uterine Fibroids

\section{Introduction}

It is estimated that infertility affects about 48.5 million couples, of which only $35 \%$ seek medical assistance [1]. Infertility is a matter for concerne in Africa given the stigmatisation attached to it and instablility in couples without a child which represents $7 \%-21 \%$ depending on the region [2]. Although infertility affects men, women, and both; female infertility is the one of the greatest challenges in contemporary gynecological practice [3]. Yet WHO reports that female infertility accounts for only $30 \%$ of cases in sub-Saharan Africa [4].

Among the causes of female infertility, tuboperitoneal factors play a predominant role, responsible for $42 \%$ to $77 \%$ of cases in Africa [5]. The tuboperitoneal factors have also been identified in western countries [6]. Indeed, the search for tuboperitoneal factors is an important step in the investigation of female infertility. It uses hysterosalpingography (HSG), hysterosalpingo-contrast-sonography (HyCoSy) and laparoscopy, generally considered the "gold standard" [7]. However, being an invasive and costly test, laparoscopy should be reserved for patients who may also benefit from the management of another pre-existing pelvic pathology [8]. In Sub-Sahara Africa, since laparoscopy is not readily available and expensive, the HSG is widely accepted as a basic tool for adequate first-line anatomical evaluation [5].

The sensitivity and specificity of the HSG are globally estimated at respectively $65 \%-81.41 \%$ and $47.8 \%-50 \%$ in the detection of tubal pathologies [9]; $92.1 \%$ and $85.7 \%$ for patency or tubal obstruction [10] and 35.5 and $81.3 \%$ for peritubular adhesions [11]. In addition, it has a concordance that varies between $38 \%$ [12] and 75\% [13] with laparoscopy for the diagnosis of tubal obstructions. The HSG is therefore a crucial examination which, in addition to the tubal balance, allows to explore the uterine cavity.

Several studies have presented the results of the HSG in the literature. In the study conducted by Ohene et al. [14] in Ghana, the main findings of the HSG were uterine fibroids (25.4\%), bilateral tubal obstruction (20.5\%), left tubal obstruction (12.5\%) and right tubal obstruction (10.6\%). In a South African study, tuboperitoneal factors were noted in $81.8 \%$ of cases, with peritubary adhesions in $5 \%$ and tubal obstructions in $27.4 \%$ of cases [15]. In Turkey, $21 \%$ of infertile women had unilateral tubal obstruction to the HSG and $12 \%$ had bilateral obstruction. Adnexal adhesions were suspected in $12 \%$ of cases [16].

To our knowledge, the HSG and the HyCoSy are the two means that allow, besides laparotomy, the exploration of the Fallopian tube and the uterine cavity in Kisangani. Since the digitization of the X-Ray department of Kisangani Uni- 
versity Hospital, we have witnessed a significant improvement in the quality of the images and a large attendance of the service for multiple indications. These include primary or secondary infertility, abortion, metrorrhagia and postoperative tubal balance of a myomectomy or tubal ligation. However, the main results recorded have never been documented. The purpose of this study is to determine the prevalence of the different lesions recorded at the HSG during the assessment of infertile women treated in Kisangani, in the North-East of the Democratic Republic of Congo (DRC). The study set also out to assess whether they present statistical differences between patients with primary infertility and those with secondary infertility.

\section{Patients and Methods}

This cross-sectional study was conducted in Kisangani, DRC, between June 2016 and December 2018. It focused on infertile patients treated at Kisangani University Hospital (KUH) and the Kisangani Angels Clinic (KAC) who had undergone an infertility assessment including HSG and who had given their oral consent to participate in the study. These two medical units were chosen because they support the vast majority of infertile couples in the City.

In this study, data collection was prospective. At admission, all patients were interviewed to get information regarding age, age of first sexual intercourse (coitarche), infertility duration, gynecological history, obstetrical history; and history of pelvic surgery including fibroidectomy, ovarian cystectomy, appendectomy, salpingectomy/salpingostomy, adhesiolysis and caesarean section. After physical examination, they had undergone an infectious investigation including inflammatory assessment, vaginal bacteriological sampling, anti-Chlamydia trachomatis serology, TPHA, VDRL and HIV serology, as well as the hormonal assessment. The HSG was scheduled between the 7th and 12th day of the menstrual cycle, and performed in the digitized room of X-ray department of the KUH. To rule out the possibility of early pregnancy, we performed a systematic plasma beta-human chorionic gonadotrophin (b-hCG) prior to any HSG procedure, although the HSG was performed during the first phase of the menstrual cycle. Pregnancy, active genital infection, the history of recent uterine surgery, genital bleeding and known allergy to iodinated contrast were considered as contraindications to the HSG. Patients with history of ectopic pregnancy or tubal surgery, those who refused to perform the HSG and patients who did not consent to the study were excluded. Finally, a total of 130 patients were included and 12 were excluded.

According to our protocol, the HSG was not performed under intravenous sedation. We are properly do an oral nonsteroidal anti-inflammatory drug (Diclofenac $50 \mathrm{mg}$ ) 30 to 45 minutes earlier to relieve pain or discomfort during and after the test, an antispasmodic (40 mg Papaverine or $10 \mathrm{mg}$ of Hyocine) one hour before the test to prevent tubal spasm, and antibiotic prophylaxis made of Doxycycline (100 to $200 \mathrm{mg}$ daily for 5 days), to start 48 hours before the test. In case of allergy to Doxycycline, we give Azythromycin for 4 days $(1 \mathrm{~g}$ on the first 
day then $500 \mathrm{mg}$ per day), starting the day before the test.

The HSG was performed by an experienced Radiologist, assisted by a Gynecologist, using the usual metal cannula system and using a Pozzi pliers. When the cannula was in place, a water-soluble iodinated contrast medium (TELEBRIX-HYSTERO $250 \mathrm{mg} \mathrm{I} / \mathrm{ml}$, GUERBET, France) was gently administered. A minimum of $5 \mathrm{X}$-rays were systematically taken, including a pelvis shot without preparation and 4 facial images (beginning of filling, face repletion, early evacuation, late peritoneal shaking) during the administration of the radiopaque product. In addition to the 5, other frontal images were taken as a function of the discharge of contrast media into the pelvic cavity, and the profile images were made only if necessary. All snapshots were read and documented by two qualified and experienced Radiologists, and the final result was obtained by consensus.

We used Epi Info ${ }^{\mathrm{TM}}$ 7.2.2.6 software to analyze the data. The frequency, the percentage, the averages and their standard deviations allowed us to describe the sample. Pearson's chi-square or Exact Fisher at the $\mathrm{p}<0.05$ significance level was used to test the association between the HSG results and the type of infertility.

Before conducting the study, the special authorizations of the KUH and KAC authorities were obtained and the protocol was approved by the Ethics Committee of the University of Kisangani. The information was collected, processed and analyzed with the strictest anonymity.

\section{Results}

During the study period, 130 HSGs were performed including 43 (33.07\%) for primary infertility and 87 (66.93\%) for secondary infertility. The age of the patients ranged from 21 to 49 years with an average of $33.52 \pm 6.01$ years (32.41 years \pm 6.78 for primary infertility and 34.06 years \pm 5.56 for secondary infertility). The history of pelvic inflammatory disease (PID) was noted in 9 patients, with a predominance for secondary infertility ( $11 \%$ vs. $4.65 \%)$. The demographic characteristics and history of these patients are presented in Table 1, depending on the type of infertility.

Table 1. Demographic characteristics and patient histories based on the type of infertility.

\begin{tabular}{cccc}
\hline Paramètre & $\begin{array}{c}\text { Frequency } \\
(\mathbf{n}=\mathbf{1 3 0})\end{array}$ & $\begin{array}{c}\text { Primary infertility } \\
(\mathbf{n}=43)\end{array}$ & $\begin{array}{c}\text { Secondary infertility } \\
(\mathbf{n}=\mathbf{8 7})\end{array}$ \\
\hline $\begin{array}{c}\text { Age }^{\star} \\
\text { Gesture }\end{array}$ & - & $32.41 \pm 6.78$ & $34.06 \pm 5.56$ \\
Parity & - & $0(0 \%)$ & $2.80 \pm 2.34$ \\
History of PID & - & $0(0 \%)$ & $1.18 \pm 1.53$ \\
History of abortion & $9(6.92 \%)$ & $2(4.65 \%)$ & $10(11 \%)$ \\
History of fibroidectomy & $29(22.30 \%)$ & $12(27.91 \%)$ & $17(19.54 \%)$ \\
History of ovarian cystectomy & $56(43.07 \%)$ & $15(34.88 \%)$ & $41(47.13 \%)$ \\
History of appendectomy & $67(51.53 \%)$ & $19(44.19 \%)$ & $48(55.17 \%)$ \\
\hline
\end{tabular}

${ }^{\star}$ Mean \pm Standard Deviation. 
The results of the HSG are shown in Table 2. Of 130 cases of the HSG, 123 (94.62\%) were pathological and 7 (5.38\%) non-pathological. The pathologies observed were dominated by tubal obstruction $(72.31 \%)$, suspicion of peritubary adhesions (10.77\%), uterine fibroids (8.46\%), uterine malformations $(6.15 \%)$ and retroverted uterus $(4.62 \%)$.

Tubal obstructions were diagnosed in $72.31 \%$ of cases $(94 / 130)$ including bilateral obstructions in $30 \%$ and unilateral obstructions in $42.3 \%$ of cases. These obstructions were associated with the left hydrosalpinx (Figure 1(a) and Figure 1 (b)) in $9.23 \%$ and right in $6.15 \%$ of cases. These tubal lesions appeared to be more frequent in patients with secondary infertility, but the difference was not significant ( $\mathrm{p}=0.35$ for obstructions, $\mathrm{p}=0.24$ for hydrosalpinx).

Uterine anomalies were observed in $25.38 \%$ of cases, mainly made of uterine fibroids, uterine malformations, retroverted uterus, uterine synechiae (Figure (b)) and uterine dextroversion (Figure 2(a)). Uterine malformations included one case of unicorn uterus, five cases of uterine hypoplasia and two cases of arched uterus (Figure 2(b)). No obvious associations were observed between these uterine anomalies and the type of infertility $(\mathrm{p}=0.19)$.

Table 2. Pathologies observed at the hysterosalpingography.

\begin{tabular}{|c|c|c|c|c|}
\hline HSG results & $\begin{array}{c}\text { Frequency } \\
(n=130)\end{array}$ & $\begin{array}{c}\text { Primary } \\
\text { infertility } \\
(n=43)\end{array}$ & $\begin{array}{l}\text { Secondary } \\
\text { infertility } \\
(\mathbf{n}=87)\end{array}$ & p-value \\
\hline Bilateral tubal obstruction & $39(30 \%)$ & $15(23.25 \%)$ & $24(27.58 \%)$ & \\
\hline Right tubal obstruction & $30(23.07 \%)$ & $10(23.25 \%)$ & $20(22.98 \%)$ & 0.35 \\
\hline Left tubal obstruction & $25(19.23 \%)$ & $7(16.27 \%)$ & $18(20.68 \%)$ & \\
\hline Bilateral hydrosalpinx & $5(3.85 \%)$ & $2(4.65 \%)$ & $3(3.45 \%)$ & \\
\hline Right hydrosalpinx & $8(6.15 \%)$ & $1(2.33 \%)$ & $6(6.89 \%)$ & 0.24 \\
\hline Left hydrosalpinx & $12(9.23 \%)$ & $3(6.98 \%)$ & $9(10.34 \%)$ & \\
\hline Uterine anomalies & $33(25.38 \%)$ & $13(30.23 \%)$ & $20(22.99 \%)$ & 0.19 \\
\hline - $\quad$ Uterine fibroids & $11(8.46 \%)$ & $5(11.63 \%)$ & $6(6.90 \%)$ & \\
\hline - $\quad$ Uterine malformations ${ }^{*}$ & $8(6.15 \%)$ & $6(13.95 \%)$ & $2(2.30 \%)$ & \\
\hline - $\quad$ Retroverted uterus & $6(4.62 \%)$ & $1(2.33 \%)$ & $5(5.75 \%)$ & \\
\hline - Uterine synechiae & $4(3.08 \%)$ & 0 & $4(4.60 \%)$ & \\
\hline - incompetent cervico isthmus & $2(1.54 \%)$ & 0 & $2(2.30 \%)$ & \\
\hline - $\quad$ Uterine dextroversion & $2(1.54 \%)$ & $1(2.33 \%)$ & $1(1.15 \%)$ & \\
\hline Peritubal adhesions & $14(10.77 \%)$ & $3(6.98 \%)$ & $11(12.64 \%)$ & 0.25 \\
\hline
\end{tabular}

Uterine malformations: 1 case of monocorn uterus, 5 cases of uterine hypoplasia and 2 cases of arched uterus. 


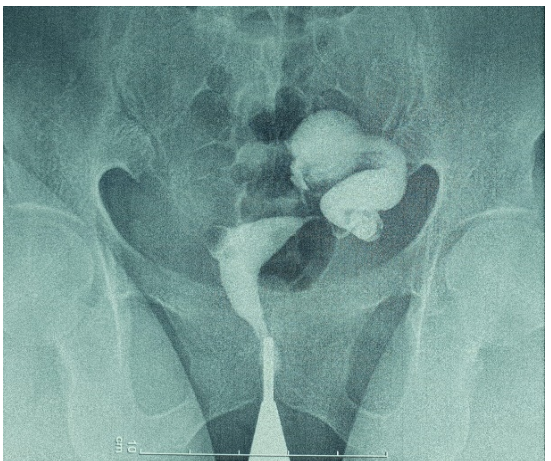

(a)

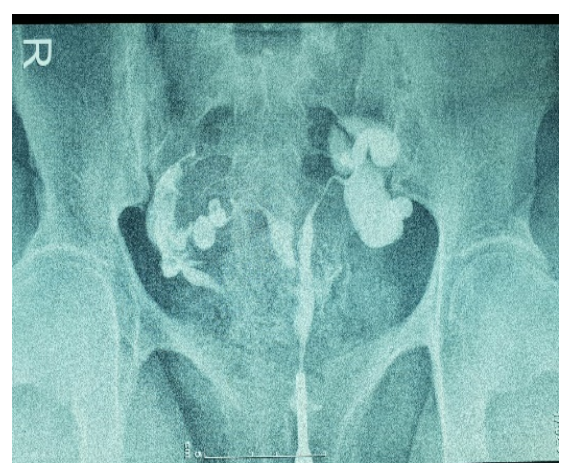

(b)

Figure 1. (a) Right proximal tubal obstruction and left hydrosalpinx in a 34-year-old nulligest with an 11-year desire for maternity; (b) Uterine synechia, left hydrosalpinx, and right tubal phimosis in a 42 -year-old patient, $\mathrm{P}_{1} \mathrm{G}_{3} \mathrm{~A}_{2}$, with a history of STI (sexually transmitted infection) and a 12 -year desire for maternity. Also to note the left tubal rigidity.

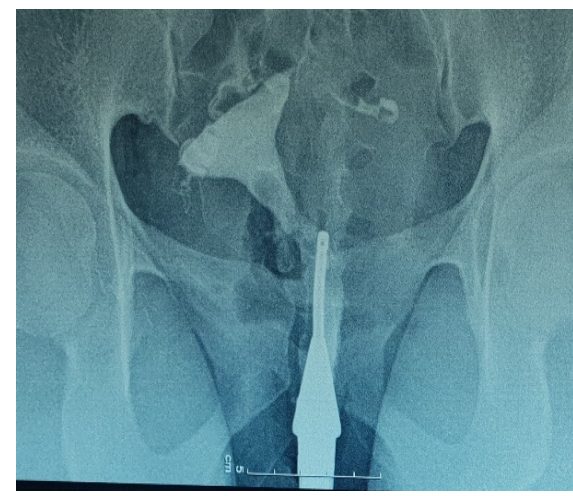

(a)

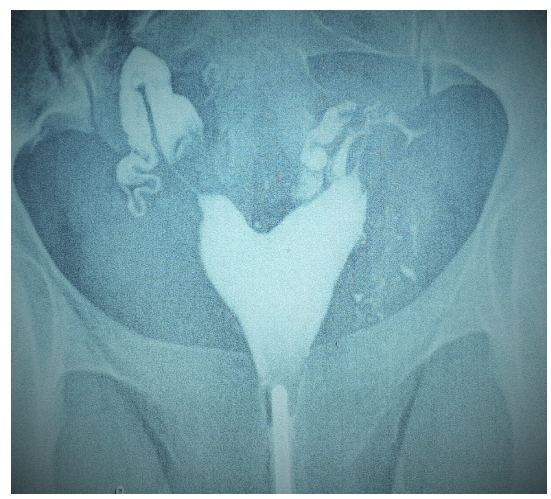

(b)

Figure 2. (a) Uterine dextroversion fixed in a 28-year-old patient, nulligest with 7-year desire for maternity; (b) Depression of the uterine fundus compatible with an arched uterus; Right distal tubal obstruction and dilatation, left tubal phimosis in a 31-year-old patient, $P_{2} G_{16} A_{14}$, with a 5-year desire for maternity.

\section{Discussion}

The HSG is a fairly common test in most imaging services in sub-Saharan Africa, reflecting the importance of this test in the management of gynecological pathology. It is mainly performed in women during periods of genital activity with a frequency peak at around 33 years, as confirmed by most studies [5] [14] [17]. In our study, the average age of sampled women was 32.41 years for primary infertility and 34.06 years for secondary infertility.

The HSG is mainly performed in a context of primary and secondary infertility [17]. While primary infertility prevails in some parts of the world, as in Iran and Turkey [16] [18], African literature describes secondary infertility as its most common form [5] [14] [19]. This was noted in our study where 87 (66.92\%) patients had secondary infertility. This high prevalence of secondary infertility in Africa is believed to be related to PID specific to sexually transmit- 
ted infections (STIs), post abortum infections and puerperal infections [14]. We recorded the antecedent of PID in $11.49 \%$ of cases in patients with secondary infertility compared to $4.62 \%$ of cases for primary infertility. Similarly, 71 patients with secondary infertility had the antecedent of abortion. These women with secondary infertility would have a high probability of having structural lesions of the Fallopian tube, compared with those with primary infertility [20]. This is why the exploration of the Fallopian tube and thus the HSG keeps a prominent place in the balance sheet of female infertility.

In our study, $94.62 \%$ of the HSG were pathological and $5.38 \%$ were non-pathological. These results are different from those of Bukar et al. in Nigeria [21] and Ohene et al. in Ghana [14] who found $70.6 \%$ and $60.3 \%$ of the HSG's pathological findings, respectively in 2011 and 2015. Guena et al. [17] performed 120 HSG in Cameroon, of which $89.17 \%$ were pathological. Conversely, Adrian et al. [22] recorded 55\% of normal HSG results in Switzerland. The analysis of these different studies proves that the pathological results of the HSG prevail in countries with limited resources. The pathologies diagnosed in our series consisted mainly of tubal obstructions (72.31\%). These obstructions were associated with hydrosalpinx in $19.23 \%$. We also observed uterine anomalies in $25.38 \%$ and peritual adherent lesions in $10.77 \%$ of cases. These lesions observed in our study correspond to the range of anomalies recorded by many other authors but to different degrees. In a series of 1140 HSGs carried out in Ghana for infertility assessment, Ohene [14] had identified tubal obstructions in $43.6 \%$ of cases and uterine anomalies in $34.5 \%$ of cases. In Cameroon, tubal lesions were noted in $45.82 \%$, uterine anomalies in $33.34 \%$ and suspicion of adhesions in 5.84\% of cases [17]. Kouamé [23] found in a series of 124 HSGs performed in Ivory Coast $50.3 \%$ of uterine diseases, $25.2 \%$ of tubal pathologies and $7 \%$ of peritoneal abnormalities.

We have found that the prevalence of tubal obstructions recorded in our study is higher than all other African series. These tubal obstructions were much more unilateral than bilateral giving the patient a chance to conceive with the permeable Fallopian tube. This result would reflect the high prevalence of STIs in the DRC, a low-income and post-armed conflict country. It should be noted that some tubal obstructions may be due to tubal spasm during injection of the contrast medium. In our study, we administered antispasmodics to all patients before performing the HSG, as recommended [13]. In addition to spasm, administration of the opaque radio product can also dislodge the mucous plug or break the intra tubal and fine peritoneal adhesions, giving images of tubal permeability while the tube was initially obstructed. These different tubal obstructions observed in our study did not show a statistical association with the type of infertility ( $\mathrm{p}=0.33)$. On the other hand, Taimoora et al. [24] reported in Oman a highly significant statistical association between unilateral tubal obstruction and type of infertility, favoring secondary infertility $(\mathrm{p}<0.0001)$.

Of uterine anomalies observed in this study, uterine fibroids accounted for $8.46 \%$ of cases. This result is lower than other studies in sub-Saharan Africa [14] [17]. However, in case of uterine fibroids, infertility can result from the effects of 
submucosal fibromyoma, the alteration of uterine contractility, which could harm the interactions between sperm and egg or embryo migration, which is seen in uteri with multiple large fibroids and significant distortion of the uterine cavity [25].

Congenital malformations of the uterus were observed in $6.15 \%$ of the HSG cases with arched uteri in $1.54 \%$ of cases. Our prevalence of congenital malformations of the uterus correlates with that of Pakistan, where congenital malformations of the uterus were observed in $6 \%$ of cases of the HSG [26]. However, the prevalence of the arched uterus is about 8 times higher than that recorded in Ghana [14] but correlates with 1.6\% reported in Uganda [27]. Retroverted uterus was diagnosed in $4.62 \%$ of the HSG cases. In a series of 807 infertile women followed by Egbase et al. [28] 38.2\% had a retroverted uterus and low implantation rate and clinical pregnancy, compared to infertile patients with anteverted uterus. In Ghana, the prevalence of the retroverted uterus is $0.4 \%$ [14].

Uterine synechiae, an abnormality characterized by a fibrous union of two sides of the uterine interior, is manifested at the HSG by persistent lacunary images despite the pressure of injection of contrast medium. We diagnosed it in $3.08 \%$ of cases. This is less than $5.84 \%$ described in Cameroon [17], but the lowest rate is reported in Ghana [14]. More than half (54.61\%) of our respondents had abortion history. These abortions, often provoked with the uterine curettage and performed under septic conditions, can explain the percentage of synechiae diagnosed in our series.

\section{Conclusion}

The HSG remains an indispensable test for investigating female infertility in resource-poor countries such as the DRC. In our study, the results of the HSG were pathological in $94.62 \%$ of cases tested, consisting mainly of tubal obstructions $(72.31 \%)$. These were much more unilateral than bilateral, and localized more to the right than to the left, and had nothing to do with a type of infertility. Tubal obstructions would reflect the high prevalence of STIs and postabortum infections as evidenced by the history of PID and abortion registered with our patients. The primary prevention of genital tract infections and the improvement of abortion practice conditions would be crucial to reduce the incidence of tubal infertility in our country.

\section{Acknowledgements}

We express our gratitude to Prof. Batina Agasa King-Salomon, Dean of the Faculty of Medicine and Pharmacy, University of Kisangani, for his valuable advice and scientific guidance, to the Management of the Kisangani University Hospital for facilitating the conduct of this study, and to the Director of Angel Clinics for allowing us to access this clinic.

\section{Conflicts of Interest}

The authors declare that they have no conflict of interest in the writing of this article. 


\section{Contribution of the Authors}

BNJD: study design, data collection, statistical analysis and data interpretation, literature review, preparation and validation of the final manuscript.

MOA: data collection, statistical analysis, substantive review, validation of the final version.

JSKV: harvest and interpretation of data, substantive review, validation of the final version.

LLG: data collection, literature review, validation of the final version.

MAA: interpretation of the data, substantive review, validation of the final version.

AKR: substantive review and validation of the final version.

KBG: design of the study, interpretation of the data, substantive review, validation of the final version, direction of the research.

\section{References}

[1] Briceag, I., Costache, A., Purcarea, V.L., Cergan, R., Dumitru, M., Sajin, M. and Ispas, A.T. (2015) Fallopian Tubes-Literature Review of Anatomy and Etiology in Female Infertility. Journal of Medicine and Life, 8, 129-131.

[2] Kalume Mushaba, A., Labama Lokwa, B. and Sergio, C.S.S. (2014) Profil clinique et étiologique de l'infertilité à Goma. Kisangani Medical, 5, 31-43.

[3] Aketayeva, A., Khamidullina, Z., Akhmetova, Z., Baubekova, A., Khismetova, Z., Dudnik, Y. and Aitbaeva, Z. (2018) Diagnosis and Treatment of Female Infertility Is One of the Major Problems in Modern Gynecology. Iranian Journal of Public Health, 47, 135-137.

[4] ORC Macro and The World Health Organization (2004) Infecundity, Infertility, and Childlessness in Developing Countries. Demographic and Health Surveys (DHS) Comparative Reports No. 9, 74 p.

[5] Ngowa, J.D.K., Kasia, J.M., Georges, N.-T., Nkongo, V., Sone, C. and Fongang, E. (2015) Comparison of Hysterosalpingograms with Laparoscopy in the Diagnostic of Tubal Factor of Female Infertility at the Yaoundé General Hospital, Cameroon. Pan African Medical Journal, 22, 264-270.

[6] Kawwass, J.F., Crawford, S., Kissin, D.M., Session, D.R., Boulet, S. and Jamieson, D.J. (2013) Tubal Factor Infertility and Perinatal Risk After. Assisted Reproductive Technology. Obstetrics \& Gynecology, 121, 1263-1271. https://doi.org/10.1097/AOG.0b013e31829006d9

[7] Lee, F.-K., Lee, W.-L. and Wang, P.-H. (2017) Is Hysterosalpingography a Good Tool to Confirm the Patency of Tubes? Journal of the Chinese Medical Association, 80, 275-276. https://doi.org/10.1016/j.jcma.2016.10.006

[8] National Institute for Health and Clinical Excellence (2013) Fertility: Assessment and Treatment for People with Fertility Problems. Nice Clinical Guideline 156-2013.

[9] Heis, M., Amarin, Z., Ibrahim, A.Y., Obeidat, N. and Omari, M. (2011) Uterine and Tubal Anatomical Abnormalities in Infertile Women: Diagnosis with Routine Hysterosalpingography Prior to Selective Laparoscopy. South African Journal of Radiology, 15, 120-122.

[10] Foroozanfard, F. and Sadar, Z. (2013) Diagnostic Value of Hysterosalpingography and Laparoscopy for Tubal Patency in Infertile Women. Nursing and Midwifery 
Studies, 1, 188-192. https://doi.org/10.5812/nms.10661

[11] Tvarijonavičienè, E. and Nadišauskienè, R.J. (2008) The Value of Hysterosalpingography in the Diagnosis of Tubal Pathology among Infertile Patients. Medicina, 44, 439-448. https://doi.org/10.3390/medicina44060057

[12] Rezk, M. and Shawky, M. (2015) The Safety and Acceptability of Saline Infusion Sonography versus Hysterosalpingography for Evaluation of Tubal Patency in Infertile Women. Middle East Fertility Society Journal, 20, 108-113. https://doi.org/10.1016/j.mefs.2014.06.003

[13] Kalume Mushabaa, A.J. (2014) Techniques modernes d'exploration de l'infertilité tubopelvienne. Kisangani Medical, 5, 59-65.

[14] Botwe, B.O., Bamfo-Quaicoe, K., Hunu, E. and Anim-Sampong, S. (2015) Hysterosalpingographic Findings among Ghanaian Women Undergoing Infertility Work-Up: A Study at the Korle-Bu Teaching Hospital. Fertility Research and Practice, 1, 9-14. https://doi.org/10.1186/s40738-015-0001-6

[15] Blumenthal, N.J., Hertzanu, Y., Ferreira, M.M.V., Mendelsohn, D.B. and Godberger, S. (1984) Hysterosalpingography in the Assessment of Infertility in Black Patients. South AfricanMedical Journal, 65, 854-856.

[16] Gokhan, G., Gamze, Y., Oznur, G., Isin, K., Lale, W. and Birol, D. (2008) Hysterosalpingography, Laparascopy or Both in the Diagnosis of Tubal Disease in Infertility. World Journal of Laparascopic Surgery, 1, 23-26.

[17] Neossi, G.M., Mbo, A.J., Moifo, B., Keugoung, B., Diallo, N.A. and Gonsu, F.J. (2014) Pratique de l'Hystérosalpingographie à l'Hôpital Régional de Ngaoundéré. Health Sciences and Diseases, 15, 1-6.

[18] Mesbazri, S., Pourissa, M., Refahi, S., Tabarraei, Y. and Dehgha, M.H. (2009) Hysterosalpingographic Abnormalities in Infertile Women. Research Journal of Biological Sciences, 4, 430-432.

[19] Adegbola, O. and Akindele, M.O. (2013) The Pattern and Challenges of Infertility Management in Lagos, Nigeria. African Health Sciences, 13, 1126-1129. https://doi.org/10.4314/ahs.v13i4.37

[20] Lash, M.M., Yaghamee, A., Strohsinitter, W. and Lalwani, S. (2008) Association between Secondary Infertility and Fallopian Tube Obstruction on Hysterosalpingography. The Journal of Reproductive Medicine, 53, 677-680.

[21] Bukar, M., Mustapha, Z., Takai, U.I. and Tahir, A. (2011) Hysterosalpingo Graphic Findings Infertile Women: A 7 Year Review. Nigerian Journal of Clinical Practice, 14, 168-170. https://doi.org/10.4103/1119-3077.84008

[22] Adrian, C.S., Nikola, F., Cornelia, U., Urech-Ruth, C. and Hohl, M.K. (2013) Kubik-Huch. Hysterosalpingography in the Work-Up of Female Infertility: Indications, Technique and Diagnostic Findings. Insights Imaging, 3, 475-483.

[23] N'goran, K., N’goan-Domoua, A.M., Konan, N., Alihonou, S., Tra-Bi, O., N'gbesso, R.D. and Kéita, A.K. (2012) Apport de l'échographie transvaginale associée à l'hystérosalpingographie dans la recherche étiologique de l'infertilité féminine à Abidjan (Côte d'Ivoire). African Journal of Reproductive Health, 16, 43-49.

[24] Al Subhi, T., Al Jashnmi, R.N., Al Khaduri, M. and Gowri, V. (2013) Prevalence of Tubal Obstruction in the Hysterosalpingogram of Women with Primary and Secondary Infertility. Journal of Reproduction \& Infertility, 14, 214-216.

[25] Carranza-Mamane, B., Havelock, J. and Hemmings, R. (2015) Prise en charge des fibromes utérins en présence d'une infertilité autrement inexpliquée. Journal of Obstetrics and Gynaecology Canada, 37, S1-S11. 
[26] Aziz, M.U., Anwar, S. and Mahmood, S. (2015) Hysterosalpingographic Evaluation of Primary and Secondary Infertility. Pakistan Journal of Medical Sciences, 31, 1188-1191. https://doi.org/10.12669/pjms.315.7545

[27] Kiguli-Malwadde, E. and Byanyima, R. (2004) Structural Findings at Hysterosapingography in Patients with Infertility at Two Private Clinics in Kampala, Uganda. African Health Sciences, 4, 178-181.

[28] Egbase, P.E., Al-sharhan and Grudzinskas, J.G. (2000) Influence of Position and Length of Uterus on Implantation and Clinical Pregnancy Rates in IVF and Embryon Transfer Treatement Cycles. Human Reproduction, 15, 1943-1946. 